International Mathematical Forum, 2, 2007, no. 46, 2283 - 2293

\title{
On $K$-Homomorphisms of $K$-Algebras
}

\author{
K. H. Dar $^{1}$ \\ Govt. College University Lahore \\ Department of Mathematics \\ Katchery Road, Lahore-54000, Pakistan \\ prof_khdar@yahoo.com \\ M. Akram \\ University College of Information Technology \\ University of the Punjab \\ Old Campus, Lahore-54000, Pakistan \\ m.akram@pucit.edu.pk
}

\begin{abstract}
The authors have introduced a class of $K$-algebras in [1] and have further extended its scope of study in literature $[2,3,4,5]$. In this paper, we introduce the notion of $K$-homomorphism of $K$-algebras, and investigate some of their properties and structure.
\end{abstract}

Mathematics Subject Classification: 06F35

Keywords: $K$-subalgebras; $K$-ideals; $K$-homomorphism; $K$-automorphism; Isomorphism

\section{Introduction}

The notion of a $K$-algebra $(G, \cdot, \odot, e)$ was first introduced by K. H. Dar and M. Akram in [1]. A $K$-algebra built on a group $(G, \cdot)$ by adjoining induced binary operation $\odot$ defined by $x \odot y=x \cdot y^{-1}$, for all $x, y \in G$, where $e$ is the identity of the group. It is attached to an abstract $K$-algebra $(G, \odot, e)$, which is non-commutative and non-associative with right identity element $e$. It is characterized by using its left and right mappings in [2]. Recently, same authors have proved that a class of $K$-algebras as a generalization of a class of $B$-algebras [13] and that of a family of $B C H / B C I / B C K$-algebras [7, 8, $9,10,11,12]$ in [4]. The notion of fuzzy structures of $K(G)$-algebras is also

\footnotetext{
${ }^{1}$ The first author is supported by HEC, Islamabad.
} 
introduced in [5]. In this paper, we discuss some properties of $K$-subalgebras and $K$-ideals in $K$-algebras in Section 2. The notion of $K$-homomorphisms of $K$-algebras is introduced, and some of its related properties are investigated in Section 3.

\section{$2 \quad K$-subalgebras}

In this section, we cite notion of $K$-algebras and extend study of $K$-subalgebra.

Definition 2.1. [1] An algebra $(G, \odot, e)$ built on a group $G$ with identity $e$ is called a $K$-algebra on $G$ (briefly, $K(G)$-algebra), if $G$ is not elementary abelian 2-group and observes the following $\odot$-axioms:

$(\mathrm{k} 1)(x \odot y) \odot(x \odot z)=(x \odot((e \odot z) \odot(e \odot y))) \odot x$,

$(\mathrm{k} 2) x \odot(x \odot y)=(x \odot(e \odot y)) \odot x$,

(k3) $x \odot x=e$,

(k4) $x \odot e=x$,

(k5) $e \odot x=x^{-1}$,

for all $x, y, z \in G$.

If the group $G$ is abelian then the axioms $(\mathrm{k} 1)$ and $(\mathrm{k} 2)$ of $K$-algebra change to be $(\overline{k 1})$ and $(\overline{k 2})$ respectively where

$(\overline{k 1})(x \odot y) \odot(x \odot z)=z \odot y$,

$(\overline{k 2}) x \odot(x \odot y)=y$,

for all $x, y, z \in G$.

Definition 2.2. A $K(G)$-algebra $(G, \odot, e)$ is abelian if, $g \odot(e \odot x)=x \odot(e \odot g)$, for all $x, g \in G$.

Proposition 2.3. $A K(G)$-algebra $(G, \odot, e)$ is abelian if and only if, $e \odot x=x$, for all $x \in G$.

Proof. Routine.

Proposition 2.4. In a $K(G)$-algebra $(G, \odot, e)$, the following equations are valid if the group $G$ is non-abelian:

$(\mathrm{k} 6)(x \odot y) \odot(u \odot v)=(x \odot(e \odot v) \odot(e \odot y)) \odot u$.

$(\mathrm{k} 7)(x \odot y) \odot z=x \odot(z \odot(e \odot y))$. 
$(\mathrm{k} 8) e \odot(e \odot x)=x$.

$(\mathrm{k} 9) e \odot(x \odot y)=y \odot x=(e \odot x) \odot(e \odot y)$.

$(\mathrm{k} 10) x \odot y=e$ if and only if $x=y$.

Proof. The proof is straightforward and we omit it.

Definition 2.5. [1] A non-empty subset $H$ of a $K$-algebra $(G, \odot, e)$ is called $K$-subalgebra if

(i) $e \in H$,

(ii) $h_{1} \odot h_{2} \in H$, for all $h_{1}, h_{2} \in H$.

Example 2.6. [1] Consider the $K\left(S_{3}\right)$-algebra $\left(S_{3}, \odot, e\right)$ on the symmetric group $S_{3}=\{e, a, b, x, y, z\}$ where $e=(1), a=(123), b=(132), x=(12)$, $y=(13), z=(23)$, and $\odot$ is given by the following Cayley table:

\begin{tabular}{c|cccccc}
$\odot$ & $e$ & $x$ & $y$ & $z$ & $a$ & $b$ \\
\hline$e$ & $e$ & $x$ & $y$ & $z$ & $b$ & $a$ \\
$x$ & $x$ & $e$ & $a$ & $b$ & $z$ & $y$ \\
$y$ & $y$ & $b$ & $e$ & $a$ & $x$ & $z$ \\
$z$ & $z$ & $a$ & $b$ & $e$ & $y$ & $x$ \\
$a$ & $a$ & $z$ & $x$ & $y$ & $e$ & $b$ \\
$b$ & $b$ & $y$ & $z$ & $x$ & $a$ & $e$
\end{tabular}

We see that $K$-subalgebra $\left(A_{3}, \odot, e\right)$ is a proper subalgebra having the following table:

\begin{tabular}{c|ccc}
$\odot$ & $e$ & $a$ & $b$ \\
\hline$e$ & $e$ & $b$ & $a$ \\
$a$ & $a$ & $e$ & $b$ \\
$b$ & $b$ & $a$ & $e$
\end{tabular}

If $H=\{e, x\}=C_{2}$ then $K\left(S_{3}\right)$-subalgebra $\left(C_{2}, \odot, e\right)$ is an improper in $K\left(S_{3}\right)$ by the Cayley table.

\begin{tabular}{c|cc}
$\odot$ & $e$ & $x$ \\
\hline$e$ & $e$ & $x$ \\
$x$ & $x$ & $e$
\end{tabular}

Remark. (1) For every subgroup $H$ of group $G$ there exists a proper $K$ subalgebra $(H, \odot, e)$ if $H$ is not an elementary abelian 2-subgroup of $G$. 
(2) If $H$ is an elementary abelian 2-subgroup of $G$ then $K$-subalgebra $(H, \odot, e)$ is improper.

Proposition 2.7. In a $K$-algebra $(G, \odot, e)$, let $g$ be a fixed element of $G$ and $H$ be a subgroup of a group $G$. Then $H_{g^{2}}=\{g \odot(g \odot x): x \in G\}$ forms a $K$-subalgebra.

Proof. Since $g \odot(g \odot e)=e \in H_{g^{2}}$, where $e \in H$ and

$$
\begin{aligned}
(g \odot(g \odot x)) \odot(g \odot(g \odot y)) & =(g \odot((e \odot y) \odot(e \odot x))) \odot g \\
& =(g \odot(e \odot(y \odot x))) \odot g \\
& =(g \odot(x \odot y)) \odot g \\
& =g \odot(g \odot(e \odot(x \odot y))) \\
& =g \odot(g \odot(y \odot x)) \in H_{g^{2}}
\end{aligned}
$$

for all $x, y \in H$ and $g \odot(g \odot x), g \odot(g \odot y) \in H_{g^{2}}$. This complete the proof.

Definition 2.8. A proper $K$-subalgebra $(H, \odot, e)$ of a $K$-algebra $(G, \odot, e)$ is called a $K$-ideal if for for all $x \in G, g \in G, x \odot g$ and $g \odot(g \odot x) \in H$ implies $x \in H$.

In example 2. 6 , the $K$-subalgebra $\left(A_{3}, \odot, e\right)$ is a $K$-ideal of $K$-algebra $(G, \odot, e)$.

Proposition 2.9. Let $H_{1}$ and $H_{2}$ be two $K$-subalgebras(K-ideals) of a $K$ algebra $(G, \odot, e)$ then

(a) $H_{1} \cap H_{2}$ is a $K$-subalgebra $(K$-ideal) of $(G, \odot, e)$.

(b) $H_{1} \odot H_{2}$ is a $K$-subalgebra $(K$-ideal) of $(G, \odot, e)$ if and only if

$H_{2} \odot H_{1}=H_{1} \odot H_{2}\left(\right.$ either $H_{1}$ or $H_{2}$ is ideal $)$.

Proof. Straightforward.

Proposition 2.10. For each proper normal subgroup $H$ of $G$ there corresponds a proper $K$ - ideal $(H, \odot, e)$ of $(G, \odot, e)$.

Proof. If $H$ is a normal subgroup of $G$ then $g x g^{-1} \in H$ for all $g \in G$ implies $x \in H$. Thus $x g^{-1}=x \odot g$ and $g \odot(g \odot x) \in(H, \odot, e)$ imply $x \in H$, which proves that $(H, \odot, e)$ is a $K$-ideal of $K$-algebra $(G, \odot, e)$.

Conversely, if $(H, \odot, e)$ is a $K$-ideal of $K$-algebra then $g \odot(g \odot x)$ and $x \odot g$ $\in H$. This implies that $g x g^{-1} \in H$ for all $g \in G$ and $x \in H$. This completes the proof

Corollary 2.11. The improper $K$-subalgebras of a $K$-algebra correspond to the improper $K$-ideals of the $K$-algebra $(G, \odot, e)$. 
Corollary 2.12. If $G$ is a simple group then the $K$-algebra is a simple algebra having no proper $K$-ideal.

Theorem 2.13. Let $K_{1}=(G, \odot, e)$ be a $K$-algebra on $G$. If $Z(G)$ is the centre of the group $G$ then $(Z(G), \odot, e)=Z(G, \odot, e)$, the centre of $K_{1}$.

Proof. From definition 2.2, if $h$ commutes with $g$ in $K_{1}$ then $g \odot(e \odot h)=$ $h \odot(e \odot g)$. If $z_{1}, z_{2} \in Z(G)$ then $z_{1} \odot(e \odot g)=g \odot\left(e \odot z_{1}\right)$ and $z_{2} \odot(e \odot g)=$ $g \odot\left(e \odot z_{2}\right)$ for all $g \in G$. Then $z_{1} \odot z_{2} \in Z\left(K_{1}\right)$ since, for all $g \in G$,

$$
\begin{aligned}
\left(z_{1} \odot z_{2}\right) \odot(e \odot g) & =z_{1} \odot\left((e \odot g) \odot\left(e \odot z_{2}\right)\right) \\
& =z_{1} \odot\left(e \odot\left(g \odot z_{2}\right)\right) \\
& =z_{1} \odot\left(z_{2} \odot g\right) \\
& =\left(z_{1} \odot(e \odot g) \odot z_{2}\right. \\
& =\left(g \odot\left(e \odot z_{1}\right)\right) \odot z_{2} \\
& =g \odot\left(z_{2} \odot\left(e \odot\left(e \odot z_{1}\right)\right)\right) \\
& =g \odot\left(z_{2} \odot z_{1}\right) \\
& =g \odot\left(e \odot\left(z_{1} \odot z_{2}\right)\right) \text { for all } g \in K_{1} .
\end{aligned}
$$

Hence $z_{1} \odot z_{2} \in Z\left(K_{1}\right)$, the centre of $K_{1}$ and

$$
(Z(G), \odot, e) \subseteq Z\left(K_{1}\right)
$$

For the converse, if $z_{1}, z_{2} \in Z\left(K_{1}\right)$, then $z_{1} \odot(e \odot g)=g \odot\left(e \odot z_{1}\right)$ and $z_{2} \odot(e \odot g)=g \odot\left(e \odot z_{2}\right)$ Thus $z_{1} \odot z_{2} \in(Z(G), \odot, e)$ since

$$
\left(z_{1} \odot(e \odot g)\right) \odot\left(z_{2} \odot(e \odot g)\right)=z_{1} \odot z_{2}=z_{1} z_{2}^{-1} \in Z(G) .
$$

Hence

$$
Z\left(K_{1}\right) \subseteq(Z(G), \odot, e)
$$

Inequalities (a) and (b) imply the equality and the proof is completed.

\section{K-homomorphisms of K-algebras}

In a $K$-algebra $K(G)=(G, \odot, e)$ which is built on a group $G$, we see that set $K(G)=$ set $G$ and hence the induced operation $\odot$ on $G$ is defined. We see easily that the mapping: endomorphisms and automorphisms of $K(G)$-algebra coincide set-wise [1] via actions on the respective group $G$.

In this section we generalize the notion of a mapping from one $K$-algebra $K_{1}=\left(G_{1}, \odot, e_{1}\right)$ into another $K$-algebra $K_{2}=\left(G_{2}, \odot, e_{2}\right)$. Thus we define a $K$-homomorphism in a usual way. 
Definition 3.1. A mapping $\psi$ from a $K$-algebra $K_{1}$ into $K_{2}$ is called a $K$ homomorphism if, for every $x_{1}, y_{1} \in K_{1}, \psi\left(x_{1} \odot y_{1}\right)=\psi\left(x_{1}\right) \odot \psi\left(y_{1}\right)$, where $\psi\left(x_{1}\right), \psi\left(y_{1}\right) \in K_{2}$.

Example 3.2. If $H=\{g \odot(g \odot x): x \in G\}$ then mapping $\psi:(G, \odot) \rightarrow(H, \odot)$ is a $K$-homomorphism where, for a fixed $g$ in $G, \psi(x)=g \odot(g \odot x)$ is a homomorphism from $(G, \odot)$ into $(H, \odot)$. It is easy to see that $\psi$ is a bijection.

Remark. (1) A $K$-homomorphism $\psi: K_{1} \rightarrow K_{2}$, from $K_{1}$ into $K_{2}$ is a group-homomorphism, from $G_{1}$ into $G_{2}$ and the vice versa.

(2) A $K$-homomorphism $\psi$ is called as usual, a monomorphism, epimorphism and isomorphism if $\psi$ is injective, surjective and bijective respectively.

(3) If a $K$-homomorphism $\psi$ from $K_{1}$ into $K_{2}$ is a $K$-isomorphism then the algebras $K_{1}$ and $K_{2}$ are called isomorphic and are written by $K_{1} \cong K_{2}$.

(4) The relation of isomorphism $(\cong)$ defined on the set of all $K$-algebras is an equivalence relation which subdivides $K$-algebras into disjoint equivalence classes.

Proposition 3.3. Let $K_{1}=\left(G_{1}, \odot, e_{1}\right)$ and $K_{1}=\left(G_{2}, \odot, e_{2}\right)$ be two $K$ algebras and $\psi \in \operatorname{Hom}\left(K_{1}, K_{2}\right)$. Then, for $x_{1}, y_{1} \in K_{1}$ and $\psi\left(x_{1}\right), \psi\left(y_{1}\right) \in K_{2}$, we conclude that:

(1) $\psi\left(e_{1}\right)=e_{2}$.

(2) $\psi(x)=\psi\left(x^{-1}\right)$.

(3) $\psi\left(e_{1} \odot x_{1}\right)=e_{2} \odot \psi\left(x_{1}\right)$.

(4) $\psi\left(x_{1} \odot x_{2}\right)=e_{2}$, if and only if $\psi\left(x_{1}\right)=\psi\left(x_{2}\right)$.

(5) If $H_{1}$ is a subalgebra of $K_{1}$ then $\psi\left(H_{1}\right)$ is a subalgebra of $K_{2}$.

(6) If $H_{1}$ is an ideal of $K_{1}$ then $\psi\left(H_{1}\right)$ is an ideal of $K_{2}$.

Proof. The proofs of (1), (2), (3) and (4) follow easily from the definition 3.1.

(5) If $x_{1}, y_{1} \in H_{1}$ then $x_{1} \odot y_{1} \in H_{1}$ as $H_{1}$ is a $K$-subalgebra of $K_{1}$ then $\psi\left(x_{1}\right), \psi\left(y_{1}\right)$ and $\psi\left(x_{1} \odot y_{1}\right) \in \psi\left(H_{1}\right)$, a subset of $K_{2} . \psi\left(H_{1}\right)$ is a $K$ subalgebra of $K_{2}$ since $\psi\left(x_{1} \odot y_{1}\right)=\psi\left(x_{1}\right) \odot \psi\left(y_{1}\right) \in \psi\left(H_{1}\right)$. By (1) it follows that $\psi\left(H_{1}\right)$ is a $K$-subalgebra of $K_{2}$.

(6) Suppose that $H_{1}$ is an ideal of $K_{1}$ and $\psi\left(e_{1}\right)=e_{2} \in \psi\left(H_{1}\right)$. Then, for all $g, x \in K_{1}, g \odot(g \odot x) \in H_{1}$ and $x \odot g \in H_{1}$ it implies that $x \in H_{1}$. Then $\psi(g) \odot(\psi(g) \odot \psi(x)) \in \psi\left(H_{1}\right)$ and $\psi(x \odot g) \in \psi\left(H_{1}\right) \rightarrow \psi(x) \in \psi\left(H_{1}\right)$, which implies that $\psi\left(H_{1}\right)$ is an ideal of $K_{2}$. 
Corollary 3.4. $\psi\left(x_{1} \odot y_{1}\right)=\psi\left(y_{1} \odot x_{1}\right)$, for all $x_{1}, y_{1} \in K_{1}$.

Corollary 3.5. $\psi\left(x_{1}\right)=\psi\left(e_{1} \odot x_{1}\right)$, for all $x_{1} \in K_{1}$.

Corollary 3.6. If $\psi: K_{1} \rightarrow K_{2}$ is an epimorphism then $\psi^{-1} \in \operatorname{Hom}\left(K_{2}, K_{1}\right)$ and the Propositions (5) and (6) hold from $K_{2}$ into $K_{1}$ under $\psi^{-1} \in$ Hom $\left(K_{2}, K_{1}\right)$.

Definition 3.7. Let $K_{1}=\left(G_{1}, \odot, e_{1}\right)$ and $K_{2}=\left(G_{2}, \odot, e_{2}\right)$ be two $K$-algebras and $\psi$ be a homomorphism from $K_{1}$ into $K_{2}$. The subset

$K e r \psi=\left\{x \in K_{1}: \psi(x)=e_{2}\right\}$ of $K_{1}$ is called the kernel of $\psi$.

We see that:

(a) For each $\psi \in \operatorname{Hom}\left(K_{1}, K_{2}\right)$ there exists $\operatorname{Ker} \psi$ which contains at least the identity element $e_{1}$ of $K_{1}$.

(b) $\operatorname{Ker} \psi=\left\{e_{1}\right\}$ if and only if $\psi$ is a monomorphism.

(c) For every $x_{1}, y_{1} \in K_{1}, x_{1} \odot y_{1} \in \operatorname{Ker} \psi$ if and only if $y_{1} \odot x_{1} \in \operatorname{Ker} \psi$.

(d) $\operatorname{Ker} \psi$ is a subalgebra of $K_{1}$.

(e) $K e r \psi$ is an ideal of $K_{1}$.

Theorem 3.8. Any two groups $G_{1}$ and $G_{2}$ build their respective isomorphic $K$ - algebras $K_{1}=\left(G_{1}, \odot, e_{1}\right)$ and $K_{2}=\left(G_{2}, \odot, e_{2}\right)$ if and only if the groups $G_{1}$ and $G_{2}$ are isomorphic.

Proof. Let $G_{1} \cong G_{2}$ and $\psi$ be an isomorphism between $G_{1}$ and $G_{2}$. Then $\psi\left(G_{1}\right)=G_{2} \cong G_{1}$ and $\psi\left(G_{1}, \odot\right)=\left(G_{2}, \odot\right) \cong\left(G_{1}, \odot\right) \rightarrow K_{1} \cong K_{2}$. The commutative property $\psi \circ \odot=\odot \circ \psi$ establishes the theorem since

$$
\begin{aligned}
(\psi \circ \odot)(x, y) & =\psi(\odot(x, y))=\psi(x \odot y) \\
& =\psi(x) \odot \psi(y) \\
& =\odot(\psi(x), \psi(y)) \\
& =(\odot \circ \psi)(x, y), \text { for every }(x, y) \in G_{1} \times G_{2}
\end{aligned}
$$

and the following diagram commutes.

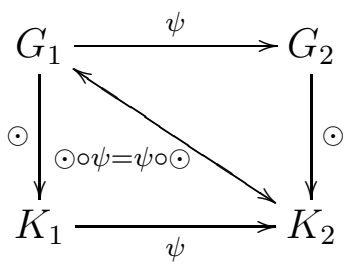


Theorem 3.9. Let $K_{1}$ and $K_{2}$ be two $K$-algebras. Let $\psi \in \operatorname{Hom}\left(K_{1}, K_{2}\right)$. A relation ' $\sim$ ' defined on $K_{1}$ by, $x \sim y$, if and only if $x_{1} \odot y_{1} \in k e r \psi$, for all $x_{1}$, $y_{1} \in K_{1}$, is an equivalence relation on $K_{1}$.

Proof. The relation $\sim$ is reflexive on $K_{1}$ by definition 2.1. The relation is symmetric by Corollary 3.5 of Proposition 3.4.

For transitivity, take $x \sim y$ and $y \sim z$. Then

$$
\begin{aligned}
& \psi(x \odot y)=e_{2}=\psi(y \odot z) \\
& \psi((x \odot y) \odot(y \odot z))=e_{2} \\
& \psi\left(\left(x \odot(y \odot z) \odot\left(e_{1} \odot y\right)\right)\right)=e_{2} \quad\left[b y K_{7}\right] \\
& \psi\left(\left(x \odot(y \odot y) \odot\left(e_{1} \odot z\right)\right)\right)=e_{2} \quad\left[b y K_{7}\right] \\
& \psi\left(\left(x \odot\left(e_{1} \odot\left(e_{1} \odot z\right)\right)\right)=e_{2} \quad\left[b y K_{3}\right]\right. \\
& \psi(x \odot z)=e_{2} \quad\left[b y K_{8}\right] \\
& x \sim z .
\end{aligned}
$$

Thus it proves the assertion.

For any homomorphism $\psi \in \operatorname{Hom}\left(K_{1}, K_{2}\right)$ there corresponds an equivalence relation on $K_{1}$ which subdivides $K_{1}$ into a set of disjoint equivalence classes whose union is the whole of $K_{1}$. Since the subdivision of $K_{1}$ is by ker $\psi$, therefore, the set of all classes is called the quotient set of $K_{1}$ by ker $\psi$ consisting of equivalence classes(cosets) denoted by

$$
\frac{K_{1}}{k e r \psi}=\left\{C_{x}: x \in K_{1}-k e r \psi\right\}
$$

where

$$
\begin{gathered}
C_{x}=k e r \psi \odot x=x \odot k e r \psi \\
C_{x} \odot C_{y}=C_{x \odot y}, \quad \text { for all } x, y \in K_{1}
\end{gathered}
$$

and

$$
C_{e_{1}}=k e r \psi \text {. }
$$

Theorem 3.10. The quotient set $\frac{K_{1}}{k e r \psi}$ consisting of all equivalence classes of $K_{1}$ by ker $\psi$ forms a $K$-algebra $\left(\frac{K_{1}}{k e r \psi}, \odot, k e r \psi\right)$, where $C_{x} \odot C_{y}=C_{x \odot y}$, for all $x, y \in K_{1}$ and $k e r \psi=e$ in the quotient algebra $\frac{K_{1}}{k e r \psi}$.

Proof. Let $C_{x}, C_{y} \in \frac{K_{1}}{k e r \psi}$ and $C_{x} \odot C_{y}=C_{x \odot y}$, for all $x, y \in K_{1}$. The properties $\left(k_{1}\right)$ to $\left(k_{5}\right)$ of definition 2.1 follow by the routine manipulation. Thus the quotient algebra $\left(\frac{K_{1}}{k e r \psi}, \odot, k e r \psi\right)$ verifies to be a $K$-algebra. 
Corollary 3.11. For each endomorphism $\psi$ on $K$-algebra $K_{1}$, the quotient algebra $\left(\frac{K_{1}}{\text { ker } \psi}, \odot, k e r \psi\right)$ is formed of order $\left|\frac{K_{1}}{k e r \psi}\right|$, if $K_{1}$ is a finite $K$-algebra.

Corollary 3.12. For each ideal $H$ of a $K$-algebra $K_{1}=(G, \odot, e)$ there exists a quotient algebra $\left(\frac{K_{1}}{H}, \odot, H\right)$ as a homomorphic image of $K_{1}$. That is, there exists a homomorphism $\theta: K_{1} \rightarrow \frac{K_{1}}{H}$, defined by $\theta(x)=x \odot H$, where

$$
\begin{aligned}
\theta(x \odot y) & =(x \odot y) \odot H \\
& =(x \odot H) \odot(y \odot H) \\
& =\theta(x) \odot \theta(y), \text { for all } x, y \in K_{1}
\end{aligned}
$$

having ker $\psi=H$. The homomorphism $\theta$ is called natural homomorphism and denoted by $n_{\theta}$.

If $\psi$ in $\operatorname{Hom}\left(K_{1}, K_{2}\right)$ is an $K$-epimorphism then:

Theorem 3.13. (Fundamental homomorphism theorem) Let $K_{1}$ and $K_{2}$ be any two $K$-algebras and $\psi$ be an epimorphism from $K_{1}$ into $K_{2}$. Then $\frac{K_{1}}{\text { ker } \psi} \cong$ $K_{2}$

Proof. Let there be a trio of $K$-algebras; $K_{1}, K_{2}$ and $\frac{K_{1}}{k e r \psi}$ for homomorphisms $\psi: K_{1} \rightarrow K_{2}$ and $n_{\theta}: K_{1} \rightarrow \frac{K_{1}}{k e r \psi}$, where $\psi$ is an epimorphism. Let $\eta$ be a mapping from $\frac{K_{1}}{k e r \psi}$ into $K_{2}$ defined by $\eta\left(C_{x}\right)=\psi(x)$ for all $x \in K_{1}$. Then $\eta$ is a homomorphism since

$$
\begin{aligned}
\eta\left(C_{x} \odot C_{y}\right) & =\eta\left(C_{x \odot y}\right)=\psi(x \odot y) \\
& =\psi(x) \odot \psi(y)=\eta\left(C_{x}\right) \odot \eta\left(C_{y}\right) \quad \forall C_{x}, C_{y} \in \frac{K_{1}}{k e r \psi}
\end{aligned}
$$

In fact, $\eta$ is an isomorphism because $k e r \eta=k e r \psi=C_{e_{1}}$ and $\psi$ is an epimorphism onto $K_{2}$. Thus

$$
\frac{K_{1}}{\operatorname{ker} \psi} \cong K_{2}
$$

Consequently, the following diagram commutes.

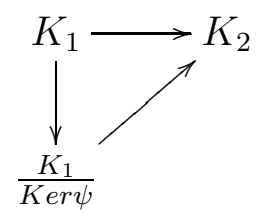




\section{Structure of homomorphisms of K-algebras}

Let there be three $K$-algebras $K_{1}, K_{2}$ and $K_{3}$. If $\phi \in \operatorname{Hom}\left(K_{1}, K_{2}\right)$ and $\psi \in$ $\operatorname{Hom}\left(K_{2}, K_{3}\right)$ then their composition $\psi \circ \phi \in \operatorname{Hom}\left(K_{1}, K_{3}\right)$ which observes the closure property if $\psi, \phi \in \operatorname{Hom}\left(K_{1}, K_{1}\right)$. The set $\operatorname{End}\left(K_{1}\right)$, consisting of all endomorphisms of $K_{1}$, forms a semigroup with the identity element under the operation of their composition (०) which is defined by the rule $(\psi \circ \phi)(x)=$ $\psi(\phi(x))$, for all $x \in X$. It is known in [1] that $\operatorname{Aut}\left(K_{1}\right)=\operatorname{Aut}(G)$ is a group structure and $K$-algebra $\left(\operatorname{Aut}\left(K_{1}\right), \odot, I d\right)$ built on $\operatorname{Aut}\left(K_{1}\right)$ is proper if the automorphisms of $K_{1}$ are not all of order 2 .

Theorem 4.1. Let $K_{1}=(G, \odot, e)$ be a $K(G)$-algebra on a group $G$. Then $\operatorname{Aut}\left(K_{1}\right)=(\operatorname{Aut}(G), \odot, I d)$ is $K$-algebra on the group $(\operatorname{Aut}(G), \circ)$, i.e., Aut $\left(K_{1}\right)=$ $(\operatorname{Aut}(G), \odot, I d)$.

Proof. If $\phi, \psi \in \operatorname{Aut}(G)$ and $\phi \circ \psi=\phi \circ \psi^{-1}$ then $\phi \odot I d=\phi, I d \odot \phi=\phi^{-1}$ and $\psi \circ \psi=I d$ confirm to the properties K4, K5 and K3 respectively of Definition 2.1. The properties $\mathrm{K} 1$ and $\mathrm{K} 2$ follow in routine computation.

Note that the homomorphic/ isomorphic $K$-algebraic models have similar structures as those of its group theoretic basis. Thus we state the following Theorems without proofs.

Theorem 4.2. (The second isomorphism theorem) Let $A$ be a $K$-subalgebra of $K$-algebra and let $B$ be a $K$-ideal of $K$-algebra. Then

(i) $B$ is a $K$-ideal of $A \odot B$,

(ii) $A \cap B$ is a $K$-ideal of $A$,

(iii) $\frac{A \odot B}{B} \cong \frac{A}{A \cap B}$.

Theorem 4.3. (The third isomorphism theorem) Let $K_{1}$ be a K-algebra having $K$-ideals $A$ and $B$ with $A \sqsubseteq B$. Then

(i) $\frac{B}{A}$ is $K$-ideal of $\frac{K_{1}}{A}$,

(ii) $\frac{K_{1}}{A} / \frac{B}{A} \cong \frac{K_{1}}{B}$.

Corollary 4.4. The $K$-algebra $(G, \odot, e)$ is simple if and only if the group $G$ is simple.

Lemma 4.5. (Zassenhaus lemma) Let $A$ and $B$ be $K$-subalgebras of a $K$ algebra $(G, \odot, e)$ and let $A_{1}$ and $B_{1}$ be $K$-ideals of $A$ and $B$ respectively. Then

(a) $A_{1} \odot\left(A \cap B_{1}\right)$ is a $K$-ideal of $A_{1} \odot(A \cap B)$,

(b) $B_{1} \odot\left(A_{1} \cap B\right)$ is a $K$-ideal of $B_{1} \odot(A \cap B)$,

(c) $\frac{A_{1} \odot(A \cap B)}{A_{1} \odot\left(A \cap B_{1}\right)} \simeq \frac{B_{1} \odot(A \cap B)}{B_{1} \odot\left(A_{1} \cap B\right)}$. 


\section{References}

[1] K. H. Dar and M. Akram, On a K-algebra built on a group, SEA Bull. Math. 29(1)(2005), 41-49.

[2] K. H. Dar and M. Akram, Characterization of a $K(G)$-algebra by self maps, SEA Bull. Math. 28(4) (2004), 601-610.

[3] K. H. Dar, M. Akram and A. Farooq, A note on left $K(G)$-algebras, SEA Bull. Math., 30 (2006).

[4] K. H. Dar and M. Akram, On subclasses of $K(G)$-algebras, Annals of University of Craiova, Math. Comp. Sci. Ser., 33 (2006).

[5] M. Akram, K. H. Dar, Y. B. Jun and E. H. Roh, Fuzzy structures of $K(G)$ algebra, SEA Bull Math. (To appear).

[6] Q. P. Hu and X. Li , On BCH-algebras, Math. Seminar Notes 11 (1983), 313-320.

[7] Q. P. Hu and X. Li, On proper BCH-algebras, Math. Japonica 30 (1985), 659-661.

[8] Y. Imai, and K. Iseki, On axiom System of propositional calculi XIV, Proc., Japonica Academy, 42(1966), 19-22.

[9] K. Iseki, An algebra related with a propositional calculus, Proc. Japan Acad. 42 (1966), 26-29.

[10] J. Meng, BCI-algebras and abelian groups, Math. Japo. 32 (1987), 693696.

[11] C. S. Hoo, BCI-algebras with condition(S), Math. Japonica 32 (1987), 749-756.

[12] J. Meng and Y. B. Jun, BCK-algebras, Kyung Moon Sa, Co., Seoul, 1994.

[13] J. Neggers and H. S. Kim, On B-algebras, Matematicki Vesnik , 54(2002), 21-29.

Received: December 31, 2005 